$\sqrt{ }$

EDITOR'S

CHOICE

\title{
Alarmingly poor performance in Chlamydia trachomatis point-of-care testing
}

\author{
Laura van Dommelen, ${ }^{1}$ Frank H van Tiel, ${ }^{1}$ Sander Ouburg, ${ }^{1,2}$ Elfi E H G Brouwers, ${ }^{3}$ \\ Peter H W Terporten, ${ }^{1}$ Paul H M Savelkoul, ${ }^{4}$ Servaas A Morré, ${ }^{1,2}$ \\ Cathrien A Bruggeman, ${ }^{1}$ Christian J P A Hoebe ${ }^{1,3}$
}

See Editorial, p 330

- An additional table is published online only. To view this file please visit the journal online (http://sti.bmj.com).

${ }^{1}$ Department of Medical Microbiology, Maastricht Infection Centre, Maastricht University Medical Centre, School for Public Health and Primary Care (CAPHRI), Maastricht, The Netherlands ${ }^{2}$ Department of Pathology, Laboratory of Immunogenetics, Section Immunogenetics of Infectious Diseases, VU University Medical Center, De Boelelaan, Amsterdam, The Netherlands

${ }^{3}$ Department of Infectious Diseases, South Limburg Public Health Service, Geleen, The Netherlands

${ }^{4}$ Department of Medical Microbiology and Infection Control, VU University Medical Center, De Boelelaan, Amsterdam, The Netherlands

Correspondence to Laura van Dommelen, Department of Medical Microbiology, Maastricht Infection Centre, Maastricht University Medical Centre, School for Public Health and Primary Care (CAPHRI), PO Box 5800, 6202 AZ Maastricht, The Netherlands;

lauravandommelen@yahoo.com

\section{Independence of} researchers All authors were independent, and worked independently from the distributors of the tests. Access to data All authors had full access to all of the data in the study and can take responsibility for the integrity of the data and the accuracy of the data analysis.

Data sharing technical appendix, statistical code, and dataset available from the corresponding author at: f.van. tiel@mumc.nl

Accepted 11 May 2010

\begin{abstract}
Background Infection by Chlamydia trachomatis (CT) is the most prevalent sexually transmitted infection (STI) world wide. The most frequently used diagnostic test for CT is a nucleic acid amplification test (NAAT), which is highly sensitive and specific. To further shorten time delay until diagnosis has been made, in order to prevent CT spread, the use of point-of-care (POC) tests may be the way forward.
\end{abstract}

Objectives The diagnostic performance of three POC tests, Handilab-C, Biorapid CHLAMYDIA Ag test and QuickVue Chlamydia test, was evaluated and compared with NAAT.

Methods All women, above the age of 16 years, attending for a consultation at an STI clinic between September 2007 and April 2008, were asked to participate. Women were asked to complete a short questionnaire and to collect six self-taken vaginal swabs (SVS). SVS 2 was used for NAAT and SVS 3 to 5 were randomised for the different POC tests. SVS 1 and 6 were used for determining quantitative CT load to validate the use of successive SVS. All POC tests were performed without knowledge of NAAT results. NAAT was used as the 'gold standard'.

Results 772 women were included. CT prevalence was $11 \%$ in our population. Sensitivities of the Biorapid CHLAMYDIA Ag test, QuickVue Chlamydia and Handilab-C test were $17 \%, 27 \%$ and $12 \%$, respectively.

Conclusions The evaluated POC tests, owing to their very low sensitivities, are not ready for widespread use. These results underline the need for good-quality assurance of POC tests, especially in view of the increased availability of these tests on the internet.

\section{INTRODUCTION}

World wide, Chlamydia trachomatis (CT) remains the most prevalent bacterial sexually transmitted infection (STI), with increased incidence in Europe over the past decade. ${ }^{1} \mathrm{CT}$ infection is, a major cause of reproductive morbidity, ${ }^{23}$ bacterial conjunctivitis in neonates, ${ }^{4}$ and may facilitate HIV transmission. ${ }^{5}$ The use of nucleic acid amplification tests (NAAT) with self-taken vaginal swabs (SVS) or urine have made CT testing more sensitive, specific and acceptable. ${ }^{6}$ Nevertheless, case finding and case recognition is hampered first, by the limited willingness of patients at risk to undergo STI testing because of fear of pelvic examination and stigmatisation, and second owing to the frequently asymptomatic nature of these infections. ${ }^{7}$ Moreover, with the use of NAAT, there is still a time delay between first consultation and treatment, usually around 1-2 weeks. ${ }^{8}$ Although some infections may resolve during this period, secondary transmission can take place and infection can progress. Therefore, a pointof-care (POC) test with proven diagnostic accuracy may well help limit the spread of and morbidity associated with CT.

Over the past few years, an increase in the availability of POC tests in drug stores and on the internet has been noticeable. In general, there appears to be a trend towards producing diagnostics, which are faster and easier to use. WHO has formulated criteria for a POC test which is adequate ${ }^{9}$ : a new STI diagnostic test should be affordable by those at risk, sensitive (sensitivity between $43 \%$ and $65 \%$ ), specific (specificity of $98 \%$ ), user-friendly, rapid and robust, equipment-free and deliverable to those in need (ASSURED criteria; http://www.who.int/std_diagnostics, accessed 14 June 2010). We have selected three widely available POC CT diagnostic tests, which might meet these criteria but which have not yet been evaluated thoroughly. We assessed laboratory performance and the potential acceptability, when used in optimal conditions compared with NAAT, to maximise POC test results before evaluation in non-laboratory and/or less developed settings. Moreover, the use of successive SVS was validated using a quantitative CT NAAT.

\section{METHODS}

Study setting, specimen collection and population Women above the age of 16 years applying for STI consultation between September 2007 and April 2008 were included in the study. The medical ethics committee of Maastricht University Medical Centre approved this study (MEC LLL06srs) and all participants signed a written consent form. At the STI clinic, each patient was asked to take six numbermarked SVS in the order of number (SVS 1 to 6). Patients were shown how to insert the vaginal swab by approximately $4-5 \mathrm{~cm}$ and with $10 \mathrm{~s}$ vaginal rotation and rubbing time and how to position the swab into each capped tube. During the consultation, demographic and behavioural data were collected and, if indicated, samples were collected for other STI diagnostics. All data and SVS were anonymised and transported to the hospital while refrigerated. Patients who tested positive for CT were treated with a single dose of $1000 \mathrm{mg}$ azithromycin. CT prevalence was expected to be $11 \%$ in this population with no loss to follow-up. ${ }^{6}$

\section{Point-of-care tests}

SVS 3 to 5 were used for the POC tests. The three POC tests that were validated were the Handilab-C 
(Zonda, Dallas, USA), Biorapid CHLAMYDIA Ag test (Biokit, S.A., Barcelona, Spain) and QuickVue Chlamydia test (Quidel Corporation, San Diego, USA). All POC tests had a CE mark and were commercially available. In order to control for possible differences in CT load in successively taken SVS, the POC tests were randomised before distribution, into SVS groups (named A, $B$ and C) with Handilab-C, Biorapid CHLAMYDIA Ag test and QuickVue Chlamydia tests being performed on SVS 3-4-5 in group A, SVS 4-5-3 in group B and SVS 5-3-4 in group C, respectively. The Handilab-C is an enzymatic test with a detection limit of 16 inclusion bodies/test (package insert). The Biorapid CHLAMYDIA Ag test and QuickVue Chlamydia test are antigen tests; the detection limit of the Biorapid CHLAMYDIA Ag test is 57-570 elementary bodies/test and the QuickVue Chlamydia should have a sensitivity of $81 \%$ when $<100$ inclusion forming units (IFU)/ml are present (package inserts).

All POC tests were stored and performed under optimal conditions in the medical microbiology laboratory, after training provided by the suppliers, and according to the manufacturers' instructions. One exception was the use of an SVS instead of an endocervical specimen with the Biorapid CHLAMYDIA Ag test and QuickVue Chlamydia test. The POC tests were performed in the medical microbiology laboratory, but the Handilab-C test was started at the STI clinic: 'fluid A' was allowed to mix with the specimen and left standing for $10 \mathrm{~min}$. After transportation to the laboratory, the swab was pushed through the foil in order to make a short contact with 'fluid B'. This procedure was discussed and supported by the manufacturer. The Handilab-C cannot be used during menstruation and the second step of the test performance must be completed within $24 \mathrm{~h}$ (definition of an 'on time' result). Both the Biorapid CHLAMYDIA Ag test and QuickVue Chlamydia test had to be performed within $72 \mathrm{~h}$ after collecting the SVS (definition of an 'on time' result). POC tests were performed and read by LvD and three fully qualified microbiological technicians. NAAT results and clinical data were linked to the POC test results no sooner than at the end of the study. Stratification by menstruation and time to test performance was therefore done retrospectively.

\section{NAAT tests}

The COBAS Amplicor CT/NG (Roche Diagnostics Systems, Basel, Switzerland) on SVS 2 was used as 'gold standard' for determining CT presence. Although the COBAS Amplicor CT/ NG is not licensed for SVS, previous studies have demonstrated no significant difference in performance between the use of SVS and that of endocervical swabs. ${ }^{10}{ }^{11}$ SVS 2 was placed in $1 \mathrm{ml}$ lysis buffer and after rotation for $10 \mathrm{~s}$ the swab was squeezed by pressing against the plastic tube and then removed. Next, $1 \mathrm{ml}$ diluent was added, mixed, centrifuged and $50 \mu \mathrm{l}$ of the supernatant was added to $50 \mu \mathrm{l}$ PCR Mix. The sample was processed further according to the standard operating procedure for CT PCR. A result of more than 10000 DNA copies was considered positive. All low positive samples between 2000 and 9999 copies of CT DNA were retested to confirm the presence of CT. Samples with repeatedly borderline $(n=1)$ or inhibited $(n=8)$ NAAT results were excluded from analysis.

For quantitative CT load determination, a real-time PCR (TaqMan assay) targeting the cryptic plasmid of CT (sensitivity of 0.01 IFU as compared with 1 IFU for the COBAS Amplicor and able to detect the recently reported Swedish variant of CT) or the human HLA was developed with Primer Express v2.0 (Applied Biosystems, Foster City, California, USA), described previously by Catsburg et al. ${ }^{12}$ Real-time PCR reactions were performed in a volume of $30 \mu \mathrm{l}$ PCR volume, consisting of TaqMan Mastermix (Applied Biosystems), $300 \mathrm{nM}$ of each primer, $150 \mathrm{nM}$ of each probe and $5 \mu \mathrm{l}$ prepared sample. Amplification and detection was performed with an ABI Prism 7000 sequence detection system (Applied Biosystems) using standard PCR conditions of the manufacturer, with 45 cycles. By using a chlamydial and a human target, the average chlamydial/ human cell load ratio, and IFU/swab were calculated. All samples were spiked with an optimal amount of internal control to validate the sample preparation as well as the RT-PCR procedure.

\section{Statistical analysis}

Sensitivity, specificity, negative (NPV) and positive (PPV) predictive values of the different POC tests compared with 'gold standard' PCR were calculated. Categorical variables were analysed with the Pearson $\chi^{2}$ test for independence and with Fisher's exact test where appropriate. Binary logistic regression was used to determine the influence of different variables (including randomisation) on the outcome of NAAT and POC tests. A $p$ value $<0.1$ was used for selecting variables and a $p$ value $<0.05$ was used to determine significant adjusted $O R$. Quantitative CT results were compared using the $t$ test for paired samples. A p value $<0.05$ was considered statistically significant. Analyses were performed with the SPSS package version 14.0

\section{Role of POC test providers}

None of the POC test providers had any role in the study design, collection or interpretation of the data or writing of the manuscript.

\section{RESULTS \\ Population and questionnaire}

Between September 2007 and April 2008, 772 women were included with a median age of 23 years (range 16-64). Over $95 \%$ of all clients filled in the questionnaire. The median age of first sexual contact was 16 years (range 6-36). The median lifetime number of sexual partners was nine (range $1->99$ ) and almost half of these contacts were considered as unsafe sexual contact. During the past 6 months, the median number of newly acquired sexual partners was three (mean 4; range 0->99). Only two out of 772 women were co-infected with Neisseria gonorrhoeae. No cases of syphilis or HIV were detected. In the month before visiting the outpatient clinic, 13\% (99/772) of the clients had used antibiotics, five of whom were CT positive with NAAT. The CT-positive clients could not recall which antibiotic they had used.

\section{POC tests compared with NAAT}

$C$ trachomatis testing by COBAS Amplicor resulted in a CT prevalence of $11 \%$ in our population ( $84 / 772$ clients). Sensitivities, specificities, NPV and PPV of the different POC tests compared with NAAT are presented in table 1. Results are presented according to time between collecting the SVS and performance of the POC test and subdivided for women with self-reported symptoms. Owing to logistical limitations, $49 \%$ of the Handilab-C results were performed in time. On time Handilab-C results are depicted for non-menstruating clients, since this test is not validated in the case of menstruation. Sensitivities of the Biorapid CHLAMYDIA Ag test, QuickVue Chlamydia test and Handilab-C were $17 \%, 27 \%$ and $12 \%$, respectively. The failure rate (meaning an invalid or missing test result) of $5 \%$ when including all Handilab-C results is mainly 
caused by presence of blood on the SVS, which hinders interpretation of the test result; self-reported menstruation was the probable cause of $85 \%(23 / 27)$ of the bloody samples. If all POC tests were included, sensitivity only decreased significantly in the QuickVue Chlamydia test. Binary logistic regression was performed using all POC test results, taking into account factors that might influence diagnostic test results ${ }^{13}{ }^{14}$ (details on the binary logistic regression are available in the supplementary online table). This assessment suggested no relevant influences.

\section{Quantitative CT NAAT results}

Quantitative CT NAAT (qNAAT) was used on 70/84 positive CT samples. The qNAAT was inhibited in six paired samples and in a single SVS 6; all other samples tested CT positive. Almost $30 \%$ of the bacterial loads were identical between the first and sixth swab taken. Higher bacterial loads were seen in SVS 1 (mean: $445678 \mathrm{IFU} / \mathrm{swab}$, median: $19410 \mathrm{IFU} / \mathrm{swab}$ : this is excluding extreme values with the Grubb test for outlier detection ${ }^{15}$ ) compared with SVS6 (mean: 29963 IFU/swab, median: $12180 \mathrm{IFU} / \mathrm{swab}$ : excluding extreme values). The CT load was $<100$ (but $>20 \mathrm{CFU} / \mathrm{ml}$ ) in one paired sample and in three single SVS 1 and two single SVS 6. Statistical analysis demonstrated no significant difference in POC test performance in relation to CT load for the different tests (data not shown). On average $14.6 \times 10^{6}$ HLA targets per swab were seen in SVS 1 (median: $5.0 \times 10^{6}$ HLA targets/swab), compared with an average of $706.7 \times 10^{6}$ HLA targets/swab in SVS 6 (median: $167.9 \times 10^{6}$ HLA targets/swab). The Grubb test was used to detect and remove outliers. The average bacterial load per cell was higher in SVS 1 than in SVS 6, probably owing to mucus removal by the immediately preceding five SVS.

\section{DISCUSSION}

The development and marketing of POC tests for CT has taken place in response to the demand for more rapid diagnosis, with the obvious goal of earlier treatment and prevention of secondary cases. In this study, three POC tests were evaluated under optimal laboratory conditions, in a population with a high CT prevalence (11\%). Overall, our data show that all POC tests perform alarmingly poorly.

A few limitations in our study should be mentioned. First, choosing patients solely from a western laboratory setting, limits direct translation of our results to other settings. However, the poor performance of POC tests in our setting is unlikely to improve under conditions with lower resources. Second, reproducibility of POC tests could not be assessed, since each swab could only be used for one POC test. Third, the
COBAS Amplicor does not detect the Swedish variant of CT (swCT or new variant nvCT) and POC test results might therefore be worse since some CT-positive samples might have been missed. The swCT, however, has been detected in The Netherlands in only one case so far and directly linked to a swCT-positive Swedish women (Morré SA, personal correspondence). ${ }^{16} 17$ Finally, for the Biorapid CHLAMYDIA Ag test and QuickVue Chlamydia test a SVS was used instead of an endocervical swab as stated in the package insert. As we have shown, the CT loads in the SVS were almost all above the detection limit of the different POC tests and statistical analysis demonstrated no significant influence of CT load on test performance. Moreover, the bacterial loads found in our study using SVS, are comparable to results found for endocervical swabs in a previous study. ${ }^{18}$

The strengths of our study are the large study population, the comparison of three POC tests in one and the same study, the experiments performed to control for CT load differences in successively taken SVS and, finally, the use of the ASSURED criteria as a reference enabling objective reviewing of results.

In our experience, all POC tests were easy to perform with respect to laboratory handling, but the Handilab-C was difficult to interpret, even after multiple tests had been carried out. In a previously published evaluation, a small-scale Norwegian study ${ }^{19}$ has already raised questions about the performance of the Handilab-C. In this study, $50 \%$ of all participating women, who were asked to perform the test themselves, were not certain how to interpret their Handilab-C result. Sixteen out of 157 participating women were CT positive with NAAT (used as 'gold standard'). The Handilab-C result was interpreted as positive by only four, and as uncertain by nine clients, which resulted in sensitivity between $25 \%$ and $57 \%$. Michel et al recently evaluated the Handilab-C in a group of 231 women (38/ 231 CT NAAT positive), again demonstrating a low sensitivity, and discussed this in view of the value of a CE mark. ${ }^{20}$

The QuickVue Chlamydia has been evaluated twice thus far. In a 1997 publication, the QuickVue Chlamydia was evaluated in a population of 724 women divided into two high-risk and one low-risk population. ${ }^{21}$ Sensitivity and specificity were on average $90.1 \%$ and $99.5 \%$, respectively, in the high-risk populations ( $n=366$, CT prevalence 14.1\%). Performance of the QuickVue Chlamydia in this study was compared with culture. Samples with false-positive QuickVue Chlamydia results, however, were retested with NAAT and added to the true positive results if found positive with NAAT. In contrast, culturenegative samples, with a negative QuickVue Chlamydia result, were not retested with NAAT despite a sensitivity of culture of

Table 1 Performance of the different point-of-care tests

\begin{tabular}{|c|c|c|c|c|c|c|}
\hline & $\mathbf{N}$ & Sensitivity, \% (95\% CI) & Specificity, \% (95\% Cl) & PPV (\%) & NPV (\%) & Failure (\%) \\
\hline \multicolumn{7}{|l|}{ Biorapid CHLAMYDIA Ag test } \\
\hline Performed within $72 \mathrm{~h}$ & 737 & 17.3 (8.8 to 25.9 ) & 93.5 (91.6 to 95.4$)$ & 23.2 & 90.9 & 1.2 \\
\hline Clients with symptoms & 359 & $17.0(6.3$ to 27.8$)$ & 92.6 (89.7 to 95.5$)$ & 25.8 & 88.1 & 0.8 \\
\hline \multicolumn{7}{|l|}{ QuickVue Chlamydia test } \\
\hline Performed within $72 \mathrm{~h}$ & 737 & 27.3 (17.3 to 37.2$)$ & $99.7(99.3$ to 100$)$ & 91.3 & 92.2 & 1.2 \\
\hline Clients with symptoms & 357 & 28.6 (15.9 to 41.2$)$ & $99.7(99.0$ to 100$)$ & 93.9 & 89.8 & 1.4 \\
\hline $\begin{array}{l}\text { Performed within } 24 \mathrm{~h} \text { in non- } \\
\text { menstruating women }\end{array}$ & 378 & $11.6(2.0$ to 21.2$)$ & 91.9 (89.0 to 94.9$)$ & 15.6 & 89.0 & 1.0 \\
\hline Clients with symptoms & 180 & $11.1(0.0$ to 23.0$)$ & 91.5 (87.1 to 95.9 ) & 18.8 & 85.4 & 0.6 \\
\hline All results & 735 & 22.5 (133 to 31.7$)$ & 88.9 (86.4 to 91.3 ) & 19.8 & 90.4 & 4.8 \\
\hline
\end{tabular}




\section{Key messages}

- Point-of-care tests (POC) could potentially diagnose Chlamydia trachomatis genital infections rapidly enough to enable the immediate start of treatment.

- The POC tests evaluated in this study are not ready for widespread use, owing to lack of sensitivity.

- Our results underline the need for good quality assurance of POC tests, especially in view of internet-based trading possibilities.

only $65 \%{ }^{22}$ Therefore, false-negative QuickVue Chlamydia test results would not have been detected, and performance of the QuickVue Chlamydia in this study has been overestimated. In 2002, a second evaluation was published comparing QuickVue Chlamydia with NAAT in two groups of 100 women. ${ }^{23}$ In the high-risk population, sensitivity and specificity were $65 \%$ and $100 \%$, respectively, with 16 women being positive with NAAT. In the low-risk population however, the sensitivity was only $25 \%(1 / 4)$. If both groups in the study by Rani et al are taken into account, the CT prevalence in their study is 10\% (20/200), which is comparable to the CT prevalence of $11 \%$ in our population. Recalculating sensitivity and specificity when using both populations of Rani et al, rendered a sensitivity of $55.0 \%$ (95\% CI $33.3 \%$ to $76.8 \%$ ) and a specificity of $100 \%$, which is not significantly different from our results.

As can be extrapolated from our results, a POC test with excellent performance may make a difference; assuming a primary CT transmission of $65 \%$ (without further transmission) when having sexual contact, ${ }^{24}$ a treatment delay of 2 weeks $^{8} 25$ and a POC test sensitivity of $100 \%$, eight additional new CT cases would have been avoided compared with NAAT. In contrast, when applying the same calculation to our data, the result is negative compared with NAAT and owing to falsepositive results, participants would have been treated unnecessary, especially in case of the Biorapid CHLAMYDIA Ag and Handilab-C test. In a recent evaluation, the Chlamydia Rapid Test showed promising results ${ }^{26}$; this POC test primarily would have detected fewer CT cases than NAAT, but owing to instant treatment prevent more CT cases, resulting in equal outcome in our model. A sensitivity of $83.5 \%$ is not sufficient to replace NAAT in a setting with minimal loss to follow-up; cost-benefit analysis therefore may determine if combining NAAT and a POC test is beneficial to avert additional CT cases.

In summary, results of this study, performed in a large population, show poorer laboratory performance of the different POC tests than has previously been described. The ASSURED criteria for POC testing including a sensitivity $43-65 \%$ and a specificity $98 \%,{ }^{9}$ are not met by any of the POC tests. The poor performance of all POC tests evaluated in our study has implications for public health, since the Handilab-C test remained commercially available via the internet (€29.95) during the entire inclusion period. The distributor has claimed a reliability of $98.15 \%$ (not further specified) on his website while, for instance, sensitivity in our study population was only $12 \%$. Our results underline the need for good quality assurance of POC tests, especially in view of their availability on the internet. ${ }^{27}$ Although excellent guidelines on CT POC test evaluation exist, ${ }^{28}$ these guidelines are regularly ignored, and thus tighter regulations are urgently needed to prevent unrestrained marketing. ${ }^{9}$ In our opinion, the CT POC tests we have evaluated, are not ready for widespread use.
Acknowledgements We thank the staff of the STI outpatient clinic of the South Limburg Public Health Service, The Netherlands for including study participants; Jolein Pleijster (VU University Medical Center, Amsterdam, The Netherlands) for excellent technical assistance while performing the bacterial load assays; and Petra Wolffs (Maastricht University Medical Centre, Maastricht the Netherlands) for her comments on molecular assays.

Funding This study was not funded. All rapid tests were kindly and unrestrictedly provided by the distributors.

\section{Competing interests None.}

Ethics approval This study was conducted with the approval of the Maastricht University Medical Centre, Maastricht, The Netherlands (MEC-06-4-004).

Contributors LvD was involved in study design, performed all point-of-care tests, was responsible for the statistical analysis and writing the manuscript; FHvT was involved in study design and contributed to critical revision and writing of the manuscript; SO was involved in statistical analysis and quantitative PCR assays; EEHGB was involved in study design, coordinated patient inclusion at the STD clinic and was involved in collection data from the STD clinic; PHWT was extensively involved in statistical analysis; PHMS designed the quantitative PCR assay; SAM was involved in study design, quantitative PCR assays and contributed to revision of the manuscript; $C A B$ was involved in study design and revision of the manuscript; CJPAH was involved in study design, supervised the staff at the STD clinic, was involved in data collection at the STD clinic and contributed to critical revision and writing of the manuscript.

Provenance and peer review Not commissioned; externally peer reviewed.

\section{REFERENCES}

1. van de Laar MJ, Morre SA. Chlamydia: a major challenge for public health. Euro Surveill 2007;12:E1-2.

2. Boeke AJ, van Bergen JE, Morre SA, et al. The risk of pelvic inflammatory disease associated with urogenital infection with Chlamydia trachomatis; literature review. Ned Tijdschr Geneeskd 2005;149:878-84.

3. Mardh PA. Tubal factor infertility, with special regard to chlamydial salpingitis. Curr Opin Infect Dis 2004;17:49-52.

4. Rours IG, Hammerschlag MR, Ott A, et al. Chlamydia trachomatis as a cause of neonatal conjunctivitis in Dutch infants. Pediatrics 2008;121:e321-6.

5. Laga M, Manoka A, Kivuvu M, et al. Non-ulcerative sexually transmitted diseases as risk factors for HIV-1 transmission in women: results from a cohort study. Aids 1993; 7:95-102.

6. Hoebe CJ, Rademaker CW, Brouwers EE, et al. Acceptability of self-taken vaginal swabs and first-catch urine samples for the diagnosis of urogenital Chlamydia trachomatis and Neisseria gonorrhoeae with an amplified DNA assay in young women attending a public health sexually transmitted disease clinic. Sex Transm Dis 2006;33:491-5.

7. Arkell J, Osborn DP, Ivens D, et al. Factors associated with anxiety in patients attending a sexually transmitted infection clinic: qualitative survey. Int J STD AIDS 2006;17:299-303.

8. Geisler WM, Wang C, Morrison SG, et al. The natural history of untreated Chlamydia trachomatis infection in the interval between screening and returning fo treatment. Sex Transm Dis 2008:35:119-23.

9. Peeling RW, Holmes KK, Mabey D, et al. Rapid tests for sexually transmitted infections (STIS): the way forward. Sex Transm Infect 2006;82(Suppl 5):v1-6.

10. Knox J, Tabrizi SN, Miller $P$, et al. Evaluation of self-collected samples in contrast to practitioner-collected samples for detection of Chlamydia trachomatis, Neisseria gonorrhoeae, and Trichomonas vaginalis by polymerase chain reaction among women living in remote areas. Sex Transm Dis 2002:29:647-54.

11. Skidmore S, Kaye M, Bayliss D, et al. Validation of COBAS Taqman CT for the detection of Chlamydia trachomatis in vulvo-vaginal swabs. Sex Transm Infect 2008:84:277-8; discussion 8-9.

12. Catsburg ASPHM, Vliet A, Algra J, et al. Development and evaluation of an internally controlled Real-Time quantitative PCR assay for the detection of Chlamydia trachomatis. In: Chernesky M, Caldwell H, Christiansen G, et al, Eds. Eleventh International Symposium on Human Chlamydial Infections. Niagara-on-the-Lake Ontario, Canada, 2006:521-4.

13. Marrazzo JM, Johnson RE, Green TA, et al. Impact of patient characteristics on performance of nucleic acid amplification tests and DNA probe for detection of Chlamydia trachomatis in women with genital infections. J Clin Microbiol 2005:43:577-84

14. Ghanem KG, Johnson RE, Koumans EH, et al. Cervical specimen order and performance measures of Chlamydia trachomatis diagnostic testing. J Clin Microbiol 2005;43:5295-7

15. Grubbs F. Procedures for detecting outlying observations in samples. Technometrics 1969:11:1-21

16. Morre SA, Catsburg A, de Boer M, et al. Monitoring the potential introduction of the Swedish Chlamydia trachomatis variant (swCT) in the Netherlands. Euro Surveill 2007;12:E9-10.

17. de Vries HJ, Catsburg A, van der Helm JJ, et al. No indication of Swedish Chlamydia trachomatis variant among STI clinic visitors in Amsterdam. Euro Surveill 2007; 12:E070208 3 
18. Michel CE, Sonnex C, Carne CA, et al. Chlamydia trachomatis load at matched anatomic sites: implications for screening strategies. J Clin Microbiol 2007;:45:1395-402.

19. Moi H. Handilab C Chlamydia for home testing is not what it claims. Tidsskr Nor Laegeforen 2007;127:2083-5.

20. Michel CE, Saison FG, Joshi H, et al. Pitfalls of internet-accessible diagnostic tests: inadequate performance of a CE-marked Chlamydia test for home use. Sex Transm Infect 2009;85:187-9.

21. Steingrimsson 0, Pawlak C, Van Der Pol B, et al. Multicenter comparative evaluation of two rapid immunoassay methods for the detection of Chlamydia trachomatis antigen in endocervical specimens. Clin Microbiol Infect 1997; 3:663-7.

22. Livengood CH 3rd, Wrenn JW. Evaluation of COBAS AMPLICOR (Roche): accuracy in detection of Chlamydia trachomatis and Neisseria gonorrhoeae by coamplification of endocervical specimens. J Clin Microbiol 2001:39:2928-32.
23. Rani R, Corbitt $\mathrm{G}$, Killough $\mathrm{R}$, et al. Is there any role for rapid tests for Chlamydia trachomatis? Int J STD AIDS 2002;13:22-4

24. Lin JS, Donegan SP, Heeren TC, et al. Transmission of Chlamydia trachomatis and Neisseria gonorrhoeae among men with urethritis and their female sex partners. $\mathrm{J}$ Infect Dis 1998;178:1707-12.

25. Fernando I, Oroz C, Steedman N, et al. Factors affecting time to treatment following diagnosis of genital Chlamydia trachomatis infection in Scottish genitourinary medicine clinics. Int J STD AIDS 2007;18:819-22.

26. Mahilum-Tapay L, Laitila V, Wawrzyniak JJ, et al. New point of care Chlamydia Rapid Test-bridging the gap between diagnosis and treatment: performance evaluation study. BMJ 2007;335:1190-4.

27. Owens SL, Arora N, Quinn N, et al. Utilising the internet to test for sexually transmitted infections: results of a survey and accuracy testing. Sex Transm Infect 2010;86:112-16.

28. Herring A, Ballard R, Mabey D, et al. Evaluation of rapid diagnostic tests: chlamydia and gonorrhoea. Nat Rev Microbiol 2006;4(Suppl 12):S41-8. 